\title{
Novedades florísticas para la provincia de Albacete II
}

Pedro Sánchez-Gómez ${ }^{1}$, Juan Francisco Jiménez ${ }^{1}$, Eduardo Picazo², José Luis Cánovas ${ }^{1}$, Antonio Catalán ${ }^{2}$ \& José Antonio López-Donate ${ }^{2}$

1 Departamento de Biología Vegetal, Área de Botánica, Facultad de Biología, Universidad de Murcia, 30100-Murcia. 2 Dirección General de Montes y Espacios Naturales. Consejería de Agricultura. Junta de Comunidades de Castilla-La Mancha. C/ Mayor, 46, 02071-Albacete.

\section{Resumen}

Correspondencia

P. Sánchez Gómez

E-mail: psgomez@um.es

Recibido: 11 julio 2014

Aceptado: 29 julio 2014

Publicado on-line: 10 septiembre 2014
Se presenta un listado de 11 especies de flora novedosas para la provincia de Albacete, la mayoría procedentes del Parque Natural de los Calares del Mundo y de la Sima, y algunas de ellas, de elevado interés biogeográfico.

Palabras clave: Corología, Flora, Albacete.

\begin{abstract}
New floristic data for the province of Albacete II

We present a list of 11 new reports of flora for Albacete province, several species are mainly located at Parque Natural de los Calares del Mundo y de la Sima, some of them of high biogeographical interest as well.
\end{abstract}

Key words: Chorology, Flora, Albacete.

\section{Introducción}

En la presente nota, continuación de una serie, se dan a conocer otras 11 novedades florísticas, todas ellas primeras citas para la provincia de Albacete, correspondientes en su mayoría al Parque Natural de los Calares del Mundo y de la Sima. En su conjunto corresponden a los resultados parciales de los trabajos de prospección botánica realizados por miembros del Grupo de Investigación E005/07 de la Universidad de Murcia, en colaboración con personal adscrito a la Dirección General de Montes y Espacios Naturales de la Consejería de Agricultura de la Junta de Comunidades de Castilla-La Mancha.

Para la revisión corológica de las especies se ha tenido en cuenta la base de datos recopilada por nuestro grupo de investigación en el sureste ibérico, así como los datos recogidos en Anthos (http://www.anthos.es). A nivel taxonómico, se han seguido básicamente los criterios de los géneros publicados en Flora iberica (Castroviejo 1986- 2013). También ha resultado una obra de consulta general Flora de Andalucía Oriental (Blanca et al. 2011).

Las coordenadas UTM dadas en este trabajo se encuentran referenciadas en el DATUM ED50. Los pliegos han sido depositados en el Herbario MUB de la Universidad de Murcia.

\section{Resultados}

Agropyron cristatum subsp. pectinatum (M. Bieb.) Tzvelev

Albacete: Molinicos, Carretera de Riópar a Elche de la Sierra, 30SWH5960, $950 \mathrm{msnm}$, taludes sobre sustrato dolomítico, 23-IX-2009, Sánchez-Gómez, Jiménez (MUB-SURESTE 113773).

Elemento de distribución mediterránea e irano-tu- 
raniana que presenta cierta preferencia por los sustratos margo-yesíferos en ambientes continentales y esteparios. Ocasionalmente se presenta sobre sustratos pobres en nutrientes de otra naturaleza. En la Península Ibérica es más abundante en el Valle del Ebro, submeseta norte y alrededores de Madrid, conociéndose algunas poblaciones del Sistema Ibérico turolense y Sierra de Baza (Granada). Especie que debe considerarse como muy rara en el ámbito de Castilla-La Mancha.

\section{Arabis alpina $\mathrm{L}$.}

Albacete: Riópar, Calar del Mundo, diaclasa próxima al refugio de los pastores, 30SWH5157, $1.450 \mathrm{msnm}$, roquedo, 2-VII-2007, Sánchez-Gómez, Jiménez, Picazo, Catalán, Gamo (MUB-SURESTE 100352, 112221).

Especie muy variable, propia de roquedos calizos. Presenta una amplia distribución eurosiberiana, llegando, con carácter finícola, a las montañas béticas orientales de Andalucía y Albacete. En Castilla-La Mancha se localiza en la localidad aquí mencionada, y en el Sistema Ibérico, en diversos puntos de la Serranía de Cuenca y enclaves próximos de Guadalajara.

\section{Eleocharis multicaulis (Sm.) Desv.}

Albacete: Yeste, Calar de la Sima, 30SWH4545, $1.350 \mathrm{msnm}$, herbazales con hidromorfía, 23-VI2010, Sánchez-Gómez, Jiménez, Picazo (MUBSURESTE 113514).

Elemento de amplia distribución en Europa y Rusia, llegando hasta el norte de África. Prefiere los suelos higroturbosos de naturaleza silícea, por lo que es más frecuente en la mitad occidental peninsular. Dada su rareza, es una especie protegida en el ámbito de Castilla-La Mancha, dentro de la categoría de "Interés Especial", conociéndose algunas localidades de los Montes de Toledo, Sierra Morena y sierras silíceas de Guadalajara. Diversas referencias a localidades levantinas suelen corresponder a confusiones con especies afines.

\section{Geum rivale L.}

Albacete: Yeste, Calar de la Sima, Cañada del Avellano, 30SWH4545, $1.250 \mathrm{msnm}$, herbazales con hidromorfía, 28-VI-2010, Sánchez-Gómez, Jiménez, Picazo (MUB-SURESTE 111455).

Elemento holártico, distribuido por la mitad norte de la Península Ibérica y sierras béticas orientales de Andalucía, llegando por la Sierra de Segura hasta los límites de la provincia de Albacete.

Lactuca perennis subsp. granatensis Charpin \& Fern. Casas

Albacete: Riópar, Arroyo de la Celadilla, proximidades de la pista que sube al Calar, 30SWH5558, $1.100 \mathrm{msnm}$, taludes pedregosos en borde del camino, 10-VI-2010, Sánchez-Gómez, Jiménez, Picazo (MUB-SURESTE 113352). Nerpio, Cuerda de la Gitana, 30SWH5813, $1.900 \mathrm{msnm}$, suelos pedregosos y gleras entre matorral almohadillado, 8-VII-2013, Sánchez-Gómez, Jiménez, Vera, Cánovas, Catalán, López-Donate (MUB-SURESTE 113739).

Endemismo bético que llega hasta las sierras más altas de Nerpio y al Calar del Mundo, como localidad más oriental conocida.

Sobre la interpretación del taxón existe cierta controversia. La tendencia actual es considerar que la especie en sentido amplio, de areal eurosiberiano y submediterráneo, presenta cierta variación clinal, que daría lugar, en sus formas extremas y humildes, a la subp. granatensis, de distribución bética. Otros autores opinan que las poblaciones béticas deben denominarse como $L$. singularis Wilmort; sin embargo, este taxón podría quedar relegado exclusivamente al ámbito nevadense.

\section{Lythrum thymifolia $\mathrm{L}$.}

Albacete: Yeste, Cueva Nevado, proximidades al Calar de la Sima, 30SWH4945, 1.240 msnm, herbazales con hidromorfía temporal, 28-VI-2010, Sánchez-Gómez, Jiménez, Picazo, Catalán (MUBSURESTE 111445, 111461). Bienservida, proximidades a Baños de Arriba, 30SWH2368, 825 msnm, prado terofítico con hidromorfía temporal, 27-V-2013, Sánchez-Gómez, Jiménez, Cánovas, Catalán, López-Donate (MUB-SURESTE 1134 70). Higueruela, Laguna del Salobralejo, 30SXI 3208, $920 \mathrm{msnm}$, borde de laguna con hidromorfía temporal, 18-VII-2013, Sánchez-Gómez, Jiménez, Cánovas, Catalán, López-Donate (MUB-SURESTE 113745).

Elemento ampliamente distribuido por el sur de Europa y norte de África. Prefiere los suelos arenosos húmedos, al igual que ocurre con otras especies del género. Aunque no es una especie endémica, forma parte de comunidades muy sensibles a la alteración del hábitat, junto a otras especies del mismo género, algunas de ellas consideradas 
como amenazadas a nivel nacional y regional.

\section{Margotia gummifera (Desf.) Lange}

Albacete: Yeste, Hueco de Tus, 30SWH4948, 950 msnm, pastizales bajo pinar en suelos silíceos, 06V-2010, Sánchez-Gómez, Jiménez, Picazo (MUBSURESTE 113744). Vianos, Pizorro Garví, 30SWH4176, 975 msnm, matorral en suelos silíceos, 13-V-2014, Sánchez-Gómez, Jiménez, Cánovas MUB-SURESTE 113774).

Elemento de distribución iberomagrebí. En la Península Ibérica, es más frecuente en la zona más occidental, de influencia iberoatlántica, sobre todo en suelos de naturaleza silícea. La población albacetense constituye el extremo oriental de distribución. Su presencia en la Sierra del Relumbrar, dentro del ámbito albacetense, es muy probable.

Pisum sativum subsp. elatius (M. Bieb.) Asch. \& Graebn.

Albacete: Riópar, Calar del Mundo, diaclasa próxima al refugio de los pastores, 30SWH5157, $1.450 \mathrm{msnm}$, zonas umbrosas y ambientes nemorales, 2-VII-2007, Sánchez-Gómez, Jiménez, Picazo, Catalán (MUB-SURESTE 100354, 1020 40). Villapalacios, Sierra del Relumbrar, 30SWH $871,780 \mathrm{msnm}$, sotobosque de encinar sobre sustrato silíceo, 20-V-2013, Sánchez-Gómez, Jiménez, Cánovas (MUB-SURESTE 113775).

Elemento de distribución mediterránea, las poblaciones presentes en ambientes forestales suelen corresponder a la var. brevipedunculatum P. H. Davis \& Meikle. En Albacete, se ha encontrado tanto en suelos de naturaleza caliza como silícea.

\section{Tuberaria lignosa (Sweet) Samp.}

Albacete: Riópar, Arroyo de la Celadilla, proximidades de la pista que sube por el arroyo, 30SWH5558, $1.090 \mathrm{msnm}$, prados en sotobosque mixto de pinar-quejigal, 9-VI-2010, Picazo, Gamo (MUB-SURESTE 113371). Riópar, Arroyo de la Celadilla, proximidades a la pista que sube por el arroyo, 30SWH5558, $1.090 \mathrm{msnm}$, prados en sotobosque mixto de pinar-quejigal, $16-\mathrm{VI}-$ 2010, Sánchez-Gómez, Jiménez, Picazo (MUBSURESTE 113239).

Elemento del Mediterráneo occidental y Canarias, ampliamente representado en la Península Ibérica, sobre todo en la parte occidental, donde son más frecuentes los suelos de naturaleza silícea; no obstante, presenta algunas poblaciones orientales y menorquinas.

Tras la propuesta de conservación del género $\mathrm{Tu}$ beraria (Dunal) Spach, aceptada en el Código Internacional de Nomenclatura Botánica, descartamos el binomen indicado en Flora iberica: Xolantha tuberaria (L.) Gallego, Muñoz Garm. \& C. Navarro.

\section{Verbascum lychnitis L.}

Albacete: Paterna del Madera, Sierra de Alcaraz, Río Endrinales, 30SWH5165, 1.360 msnm, cultivos abandonados, 02-VII-2008, Sánchez-Gómez, Jiménez, Picazo (MUB-SURESTE 113284).

Elemento de amplia distribución europea que llega a las montañas más húmedas del norte de África. En la Península Ibérica se distribuye sobre todo en la mitad norte, llegando algunas poblaciones aisladas a las sierras subbéticas. La localidad aquí mencionada, sirvió para la indicación de su presencia en la provincia de Albacete en Flora iberica, quedando constancia detallada de la localidad en esta publicación.

Verbascum $\boldsymbol{x}$ longiracemosum Chaub. ex St. Amans (V. sinuatum L. $\times$ V.thapsus L.)

Albacete: Riópar, camino a los Chorros, pista del retén forestal, 30SWH4857, $1.080 \mathrm{msnm}$, margen de camino, 30-VIII-2010, Sánchez-Gómez, Jiménez (MUB-SURESTE 113471, 113472, 113473).

Se ha encontrado un individuo en el margen de un camino asfaltado, entre los parentales.

\section{Agradecimientos}

Sirva este trabajo como homenaje a Pedro Gamo $(† 2011)$, inestimable compañero y colaborador en las prospecciones realizadas durante los últimos años en este territorio. Trabajo financiado parcialmente a través de un Convenio de Colaboración Científica entre la Universidad de Murcia y la entidad Gestión Ambiental de Castilla-La Mancha (GEACAM): "Conocimiento de la vegetación, los tipos de Hábitats, y la distribución de Flora amenazada en espacios de la Red Natura 2000 de la provincia de Albacete" y del proyecto CGL201130099 del Ministerio de Economía y Competitividad. 


\section{Referencias}

Anthos. 2014. Sistema de información de las plantas de España. Real Jardín Botánico, CSIC-Fundación Biodiversidad. Disponible en http://www.anthos.es (accedido en junio de 2014)
Blanca G., Cabezudo B., Cueto M., Salazar C. \& Morales Torres C. 2011. Flora Vascular de Andalucía Oriental. Universidades de Almería, Granada, Jaén y Málaga, Granada. $1751 \mathrm{pp}$.

Castroviejo, S. 1986-2013. Flora iberica 1- 8, 10-15, 1718, 20-21. Real Jardín Botánico, CSIC, Madrid. 